\title{
Perivascular fibrosis and IgG4-related disease: a case report
}

\author{
S. Monti ${ }^{1}$, G. Crepaldi ${ }^{1}$, A. Peri ${ }^{2}$, A. Pietrabissa ${ }^{2}$, P. Morbini ${ }^{3}$, \\ F. Bobbio-Pallavicini' ${ }^{1}$, C. Montecucco' ${ }^{1}$, R. Caporali ${ }^{1}$ \\ UU.O. Reumatologia, Università di Pavia, IRCCS Fondazione Policlinico S. Matteo, Pavia; \\ ${ }^{2}$ U.O. Chirurgia Generale, Università di Pavia, IRCCS Fondazione Policlinico S. Matteo, Pavia; \\ ${ }^{3}$ U.O. Anatomia Patologica, Università di Pavia, IRCCS Fondazione Policlinico S. Matteo, Pavia, Italy
}

\begin{abstract}
SUMMARY
Immunoglobulin G4-related disease (IgG4-RD) is a newly recognized fibroinflammatory condition which can potentially involve any organ. Some characteristic histopathologic features with lymphoplasmacytic infiltrate, an increased number of IgG4+ cells, storiform fibrosis and obliterative phlebitis are the mainstay for diagnosis. Serum IgG4 levels often increase. We report the case of a patient with perivascular fibrotic lesions involving the aortic arch and the splenic hilum, with a surgical biopsy-proven diagnosis of IgG4-related disease. The patient is now undergoing a low-dose corticosteroid maintenance therapy without evidence of new localizations of the disease. This case highlights the need for increasing awareness and recognition of this new, emerging clinical condition.
\end{abstract}

Key words: Immunoglobulin G4-related disease, Perivascular fibrosis, Retroperitoneal fibrosis, Aortic aneurysm.

Reumatismo, 2014; 66 (3): 245-248

\section{INTRODUCTION}

TgG4-related disease (IgG4-RD) is a newly recognized fibroinflammatory pathological entity encompassing several conditions previously regarded as separate diseases, such as chronic sclerosing sialadenitis (Küttner's) tumor, Riedel thyroiditis, Mikulicz's disease, type 1 autoimmune pancreatitis (AIP), potentially involving any organ (1).

IgG4-RD shows characteristic histopathologic features with lymphoplasmacytic infiltrate, an increased number of IgG4+ cells, storiform fibrosis, obliterative phlebitis and, frequently, mild tissue eosinophilia (2).

Serum IgG4 may be elevated, yet this is not essential for the diagnosis, since a significant proportion of patients with a confirmed histological diagnosis have normal serum levels (3).

Patients with IgG4-RD usually show a prompt response to glucocorticoids, while new evidence for the role of immunosuppressant therapies is emerging.

\section{CASE REPORT}

S.M., a 54-year-old woman, was admitted to our department with diffuse arthromyalgia, xerostomia, xerophtalmia, asthenia and mild dyspnea. She had been recently diagnosed with an undifferentiated connective tissue disease (UCTD) on the basis of her clinical picture and autoantibodies findings characterized by the positivity of antinuclear antibodies 1:320 with a nucleolar pattern, anti-Ro and anti-Scl70 positivity. Relevant medical history included favism without recent episodes of hemolytic crisis, pituitary adenoma and chronic lymphocytic thyroiditis.

One year prior to presentation, the patient had undergone cardiac surgery with prosthetic replacement of the aortic arch due to the incidental finding, during a routine echocardiography, of a periaortic mass, confirmed by thoracic computed tomography (CT) (Fig. 1A). Histology revealed perivascular fibrous connective tissue cuffing with lymphoplasmacytic infiltrate. Amyloid deposits were excluded. The
Corresponding author:

Sara Monti

Policlinico S. Matteo

Pz.le Golgi, 3 - 27100 Pavia, Italy

E-mail: sara.saramonti@gmail.com 


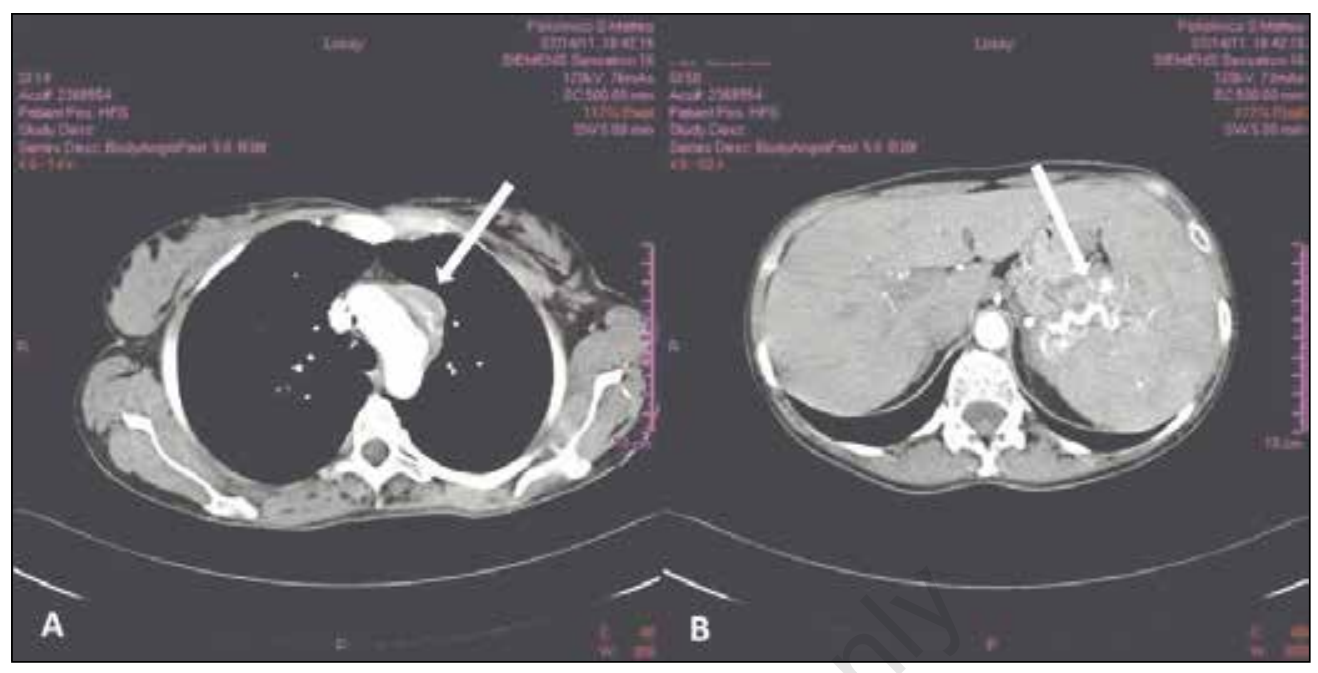

Figure 1 - Thoracic and abdominal contrast enhanced-computed tomography. A) Perivascular ulcerative mass involving the aortic arch (arrow). B) Solid lesion with calcifications completely surrounding the splenic artery, with thrombosis of the splenic vein and a moderately enlarged spleen, extending to the pancreatic tail (arrow).

etiology of the perivascular mass had not been further investigated and the patient had no signs of recurrence at the time of admission to our unit. The diagnosis of UCTD was confirmed, with a stable clinical course. Nailfold capillaroscopy did not reveal any alteration. Schirmer's test and sialometry were within normal ranges. Pulmonary function tests showed a mild, isolated, reduction of the carbon monoxide transfer factor. Echocardiography was stable, with preserved ejection fraction, no valvular defects and normal estimated systolic pulmonary artery pressure.

High resolution CT excluded an interstitial lung disease. Inflammatory markers were not raised and only a mild leukopenia (white blood cells count $3 \times 10^{3} / \mu \mathrm{L}$, with normal differential) was detected with blood tests. The clinical examination revealed a palpable, enlarged spleen with inconstant, dull pain in the upper left abdominal quadrant. An ultrasound examination showed the presence of a perivascular mass surrounding the splenic hilum. An abdominal contrast enhanced-CT disclosed a solid lesion with calcifications of $71 \times 81 \mathrm{~mm}$, extending to the pancreatic tail and lacking a clear cleavage with the organ parenchyma and no significant contrast enhancement. The splenic artery was completely sur- rounded by the mass, with thrombosis of the splenic vein and a moderately enlarged spleen (Fig. 1B). Given the striking similarities with the periaortic mass that had been previously removed, a diagnosis of IgG4-RD was hypothesized. The history of pituitary adenoma and chronic lymphocytic thyroiditis could also be part of the spectrum. Serum IgG4, even if not essential for the diagnosis, were evaluated and resulted within normal ranges $(599 \mathrm{mg} / \mathrm{L}$; normal values $80-1400 \mathrm{mg} / \mathrm{L}$ ). A course of steroids $0.5 \mathrm{mg} / \mathrm{kg}$ was started, but it did not result in a significant reduction of the perisplenic mass. A surgical procedure of distal splenopancreasectomy was agreed with the patient for a better diagnostic definition. The histological analysis revealed perivascular fibrous connective tissue with calcifications and diffuse IgG4+ lymphoplasmacitic inflammatory infiltrate, with IgG4+ >20/high power field (HPF) and a clearly increased IgG4+/IgG ratio, consistent with retroperitoneal fibrosis. A new examination of the aortic histological specimen confirmed the similarities between the two perivascular lesions, corroborating the diagnosis of IgG4-RD. The patient is undergoing a low-dose corticosteroid therapy without evidence of new localization of the disease. 


\section{DISCUSSION}

IgG4-RD is a newly recognized systemic disorder affecting a wide range of organ systems and unifying several conditions previously regarded as separate pathological entities. Since the first reports of extrapancreatic involvement and elevated IgG4 concentrations in AIP $(4,5), \operatorname{IgG} 4-\mathrm{RD}$ has been found to potentially affect every organ, including the retroperitoneum, kidneys, gastrointestinal tract, bile ducts, liver, thyroid, salivary glands, arteries, lymphnodes, skin, central nervous system, lungs, prostate and breast $(1,2)$. Clinically the disease manifests itself sub-acutely, with tumorous swelling and different grades of fibrosis of the affected sites. Multi-organ manifestations can be synchronous or follow a metachronous course (1). Highly specific histopathological features are the mainstay for diagnosis, characterized by lymphoplasmacytic infiltrate, an increased number of IgG4+ cells >10/HPF and with a IgG+/ IgG4+ ratio $>40 \%$, storiform fibrosis, obliterative phlebitis and, frequently, mild tissue eosinophilia (2). Comprehensive diagnostic criteria for IgG4-RD proposed a serum $\mathrm{IgG} 4$ cutoff of $135 \mathrm{mg} / \mathrm{dL}$ (2) to be significant. Our patient did not present with an elevation of IgG4 serum levels. However, the diagnostic utility of serum IgG4 concentrations has been recently discussed. It is well-established that a relevant proportion of patients (up to $40 \%$ ) with biopsy-proven IgG4-RD have normal serum IgG4 concentrations (3). Elevated serum IgG4 have also been described in a number of different conditions, lacking diagnostic histopathological features of IgG4-RD, such as pancreatic cancer, cholangiocarcinoma, primary sclerosing cholangitis, Castleman's disease, systemic vasculitides (6, 7). Serum IgG4 concentrations should be regarded as a useful test for the diagnosis, but have a poor specificity and a low positive predictive value in identifying patients with IgG4-RD (6). Another explanation for underestimated, false negative serum IgG4 concentrations has been recently identified in the so-called prozone effect leading to unreliable measurements of IgG4 by neph- elometry assay that occurs when samples are not adequately diluted, in the setting of extremely high IgG4 serum concentrations (7). A consistent proportion of aortitis cases have been regarded as IgG4-related. Several authors reported that IgG4-RD accounts for up to 7-9\% of all cases of non-infectious thoracic aortitis $(8,9)$. Increasing evidence of a strict relationship between retroperitoneal fibrosis (RPF), inflammatory abdominal aortic aneurysm and IgG4$\mathrm{RD}$ is emerging (10). The wide spectrum of IgG4-RD actually includes a substantial proportion of patients with idiopathic RPF (11); distinctive characteristics are typical histopathological features of IgG4-RD, middle-aged and male predominance, a history of atopy, more frequent systemic symptoms and involvement of other organs $(10,11)$. IgG4-RD usually shows a prompt response to glucocorticoids. In our case a dramatic response to steroids was not achieved; refractory cases of IgG4-RD have been reported and can be influenced by the degree of fibrosis within the affected tissue at the time of diagnosis $(1,12)$. Efficacy of immunosuppressants (azathioprine, mycophenolate mofetil, and methotrexate) as steroid-sparing agents and as remission maintenance therapy has been reported in small case series $(12,13)$. Promising and swift clinical responses have been achieved with B-cell depletion too $(1,12,14,15)$. Rituximab has been proved particularly effective in targeting the short-lived cells responsible for the specific production of IgG4 subclasses of immunoglobulins (14, 15). For selected cases, such as unstable aneurysmal aortic or large vessels lesions, surgery may still be necessary (13).

Our case emphasizes the importance of increasing the awareness and recognition of an emerging clinical condition that can potentially involve every medical field.

\section{REFERENCES}

1. Perez Alamino R, Espinoza LR, Zea AH. The great mimicker: IgG4-related disease. Clin Rheumatol. 2013; 32: 1267-73.

2. Umehara H, Okazaki K, Masaki Y, Kawano M, Yamamoto M, Saeki T, et al. Comprehen- 
sive diagnostic criteria for IgG4-related disease (IgG4-RD), 2011. Mod Rheumatol Jpn Rheum Assoc. 2012; 22: 21-30.

3. Sah R, Chari S. Serologic issues in IgG4related systemic disease and autoimmune pancreatitis. Curr Opin Rheumatol. 2011; 23: 108-13.

4. Kamisawa T, Egawa N, Nakajima H. Autoimmune pancreatitis is a systemic autoimmune disease. Am J Gastroenterol. 2003; 98: 28112.

5. Hamano H, Kawa S, Horiuchi A, Unno H, Furuya $\mathrm{N}$, Akamatsu $\mathrm{T}$, et al. High serum IgG4 concentrations in patients with sclerosing pancreatitis. N Engl J Med. 2001; 344: 732-8.

6. Carruthers MN, Khosroshahi A, Augustin T, Deshpande V, Stone JH. The diagnostic utility of serum IgG4 concentrations in IgG4-related disease. Ann Rheum Dis. 2014. [Epub ahead of print]

7. Khosroshahi A, Cheryk LA, Carruthers MN, Edwards JA, Bloch DB, Stone JH. Brief report: spuriously low serum IgG4 concentrations caused by the prozone phenomenon in patients with IgG4-related disease. Arthritis Rheumatol. 2014; 66: 213-7.

8. Stone JH, Khosroshahi A, Deshpande V, Stone JR. IgG4-related systemic disease accounts for a significant proportion of thoracic lymphoplasmacytic aortitis cases. Arthritis Care Res. 2010; 62: 316-22.
9. Koo BS, Koh YW, Hong S, Kim YJ, Kim Y-G, Lee C-K, et al. Frequency of immunoglobulin G4-related aortitis in cases with aortic resection and their clinical characteristics compared to other aortitises. Int J Rheum Dis. 2014; 4: 420-4.

10. Kasashima S, Zen Y. IgG4-related Inflammatory abdominal aortic aneurysm, spectrum of IgG4-related chronic periaortitis. Ann Vasc Dis. 2010; 3: 182-9.

11. Khosroshahi A, Carruthers MN, Stone JH, Shinagare S, Sainani N, Hasserjian RP, et al. Rethinking Ormond's disease: "idiopathic" retroperitoneal fibrosis in the era of IgG4-related disease. Medicine (Baltimore). 2013; 92: 82-91.

12. Stone JH, Zen Y, Deshpande V. IgG4-related disease. N Engl J Med. 2012; 366: 539-51.

13. Monach PA. IgG4-related disease: 2013 update. Curr Treat Options Cardiovasc Med. 2013; 15: 214-23.

14. Khosroshahi A, Carruthers MN, Deshpande V, Unizony S, Bloch DB, Stone JH. Rituximab for the treatment of IgG4-related disease: lessons from 10 consecutive patients. Medicine (Baltimore). 2012; 91: 57-66.

15. Khosroshahi A, Bloch DB, Deshpande V, Stone JH. Rituximab therapy leads to rapid decline of serum IgG4 levels and prompt clinical improvement in IgG4-related systemic disease. Arthritis Rheum. 2010; 62: 1755-62. 